\title{
Junior doctors' knowledge of clinical nutrition
}

\author{
L. Camburn and A. Forbes \\ University College London Hospitals NHS Foundation Trust, 235 Euston Road, London NW1 2BU, UK
}

\begin{abstract}
Malnutrition risk has repeatedly been identified in around $40 \%$ of hospital patients ${ }^{(1)}$ and although recent BAPEN (British Association for Parenteral and Enteral Nutrition) data suggest a modest improvement, the failure to recognise malnutrition and the need to take action remain common. Up to $70 \%$ of malnourished patients still enter and leave hospital without the condition being addressed ${ }^{(2)}$. Effective nutrition screening has been compromised by many factors, one obstacle being that the patients at highest risk are often those who are most difficult to weigh. To try and overcome both this and other problems the Malnutrition Universal Screening Tool (MUST) was devised. In validation studies it was found that although as few as $56 \%$ of patients could be weighed, all could be screened with MUST and that those identified to be at high risk had greater mortality and longer hospital stays than those at low risk ${ }^{(3)}$. In 2006 the National Institute for Clinical Excellence recommended that all hospital patients should be screened and monitored regularly for malnutrition. The role and responsibility of the doctor in managing nutrition has also been recognised in publications by the General Medical Council and the Royal College of Physicians.

The questionnaire was designed to assess the junior doctor's knowledge in assessing malnutrition risk and in using tools such as MUST. The questionnaire included clinical nutrition scenarios that junior doctors may face whilst on duty on the wards, including the recognition and management of refeeding syndrome and of line sepsis in a patient receiving parenteral nutrition. The questionnaire comprised sixteen questions with a mixture of short-answer and true or false questions yielding a maximum possible score of 25 . Participants were also asked to rate their confidence dealing with complications of parenteral nutrition and to assess the teaching received as an undergraduate. The questionnaire was completed by twenty-five doctors at a general acute teaching hospital in which MUST was adopted as the screening tool about 12 months beforehand, including six in foundation year 1, eleven in foundation year 2, four in specialist training years 1-3 and four specialist registrars. A range of specialties was represented and those currently on the nutrition firm were excluded.

Only $28 \%$ knew that MUST is a nutritional screening tool, compared with $76 \%$ who correctly reproduced the formula for calculating BMI. Sixteen (64\%) participants identified phosphate as important in the refeeding syndrome, but only ten (40\%) knew that it would be likely to be low. Seven doctors were correctly able to offer two factors that would predispose to refeeding syndrome, seven offered one factor, but eleven $(44 \%)$ were unable to list any and only three could make any relevant comment on its underlying mechanism. Perhaps unsurprisingly, therefore, scores for the management of suspected refeeding syndrome were also poor, with only three doctors obtaining maximum marks and eleven (44\%) obtaining none. Seniority did not appear to improve performance.

Participants rated their confidence in clinical nutrition at an average of only 1.9 on a scale of 1 to 5 (5 being very confident). Only $12 \%$ felt that the clinical nutrition teaching they had received at medical school was adequate.

In summary, junior doctors performed poorly in a simple test where high scores should have been expected and managed an overall average score of only $45.6 \%$. While these results are obviously disappointing, it is encouraging that participants recognised their limitations and wanted to learn. It is also encouraging that the BMI definition was well known, given that it forms an identified part of the undergraduate curriculum; this suggests that a similar emphasis on other aspects important in nutrition (such as the implementation of nutrition screening or management of refeeding syndrome) might also be successfully conveyed with their more formal inclusion within undergraduate and postgraduate training.
\end{abstract}

1. McWhirter JP \& Pennington CR (1994) Br Med J 308, 945-948.

2. Kelly IE, Tessier S, Cahill A et al. (2000) Q J Med 93, 93-98.

3. Stratton RJ, King CL, Stroud MA et al. (2006) Br J Nutr 95, 325-330. 